\title{
Two-headed butterfly vs. mantis: do false antennae matter?
}

\author{
Tania G López-Palafox ${ }^{1}$, Carlos R Cordero ${ }^{\text {Corresp. } 2}$ \\ ${ }^{1}$ Posgrado en Ciencias Biológicas, Universidad Nacional Autónoma de México, Ciudad de México, México \\ 2 Departamento de Ecología Evolutiva, Instituto de Ecología, Universidad Nacional Autónoma de México, Ciudad de México, México \\ Corresponding Author: Carlos R Cordero \\ Email address: crafaelcm@gmail.com
}

The colour patterns and morphological peculiarities of the hindwings of several butterfly species result in the appearance of a head at the rear end of the insect's body. Although some experimental evidence supports the hypothesis that the "false head" deflects predator attacks towards the rear end of the butterfly, more research is needed to determine the role of the different components of the "false head". We explored the role of hindwing tails (presumably mimicking antennae) in predator deception in the "false head" butterfly Callophrys xami. We exposed butterflies with intact wings and with hindwing tails experimentally ablated to female mantises (Stagmomantis limbata). We found no differences in the number of butterflies being attacked and the number of butterflies escaping predation between both groups. However, our behavioural observations indicate that other aspects of the "false head" help C. xami survive some mantis attacks, supporting the notion that they are adaptations against predators. 


\section{Two-headed butterfly vs mantis: do false antennae matter?}

2

3 Tania G. López-Palafox ${ }^{1}$

4 Carlos Cordero $^{2}$

5

$6{ }^{1}$ Posgrado en Ciencias Biológicas, Universidad Nacional Autónoma de México, Ciudad de

7 México, México

$8 \quad{ }^{2}$ Departamento de Ecología Evolutiva, Instituto de Ecología, Universidad Nacional Autónoma

9 de México, Ciudad de México, México

10

11 Corresponding author

12 Carlos Cordero, cordero@ecologia.unam.mx 


\section{ABSTRACT}

15 The colour patterns and morphological peculiarities of the hindwings of several butterfly species result in the appearance of a head at the rear end of the insect's body. Although some experimental evidence supports the hypothesis that the "false head" deflects predator attacks towards the rear end of the butterfly, more research is needed to determine the role of the different components of the "false head". We explored the role of hindwing tails (presumably mimicking antennae) in predator deception in the "false head" butterfly Callophrys xami. We exposed butterflies with intact wings and with hindwing tails experimentally ablated to female mantises (Stagmomantis limbata). We found no differences in the number of butterflies being attacked and the number of butterflies escaping predation between both groups. However, our behavioural observations indicate that other aspects of the "false head" help C. xami survive some mantis attacks, supporting the notion that they are adaptations against predators.

\section{INTRODUCTION}

Butterfly wings are canvases on which evolution designs solutions to the problems posed by thermoregulation, sexual selection and predation (Monteiro \& Prudic, 2010; Kemp \& Rutowski, 2011). These adaptations frequently involve compromises between selective pressures when optimal trait values differ between functions (Ellers \& Boggs, 2003), although sometimes they coincide (Finkbeiner et al., 2014). Several butterfly species exhibit colour patterns and morphological peculiarities in their hindwings that suggest, at least to the human eye, that a butterfly resting with its wings closed possess a second head at the rear end of its body (Robbins, 1980; Cordero, 2001). This appearance is enhanced by peculiar behaviours, such as the back and forth movements of the closed hindwings that presumably permit the "false antennae"— the 
37 "tails" frequently present in the border of the anal angle of the hindwings (Fig. 1a)—mimic the

38 movements of real antennae (Robbins, 1980; López-Palafox et al., 2015). False head butterflies

39

40

41

42

43

44

are especially common among the subfamily Theclinae (Lycaenidae). Several specific

hypotheses on the function of the "false head" have been advanced; all of them consider visually oriented predators as the main selective pressure, and avoidance or deflection of attacks as the main advantage (Robbins, 1980; Cordero, 2001). Although false head butterflies are textbook examples of anti-predator adaptations (e.g. Wickler, 1968; Ruxton et al., 2004), to the best of our knowledge, there are only two published experimental studies testing the effect of false heads on probability of predation in live butterflies.

Sourakov (2013) exposed two Calycopis cecrops (Lycaenidae) butterflies, a species with false head, and thirteen individuals from eleven species of butterflies and moths without false heads, to one individual predatory salticid spider (Phidippus pulcherrimus). The spider repeatedly failed to trap the lycaenid butterflies because it directed all its attacks towards the false head, but captured all individuals from the other species, mostly (11 out of 13 cases) in the first or second attack. Wourms \& Wasserman (1985) added artificial "false heads" to Pieris rapae (Pieridae) butterflies by attaching tails ("false antennae") and painting spots ("false eyes") on the anal angle of the hindwings, as well as by painting lines converging on the anal angle, three of the main components of false heads identified by Robbins (1980). Wourms \& Wasserman (1985) compared predation rates by Blue Jays (Cyanocitta cristata) between intact butterflies and butterflies with false heads added. All control and experimental butterflies attacked were caught, but the percentage of butterflies escaping during handling was twice as large in the treatment with artificial false heads as in the control group (16 out of $60 \mathrm{vs} .10$ out of 79 , respectively). The authors mention that butterflies escaped due to "mishandlings" by the 
60 birds, i.e. due to errors resulting from misdirected strikes while handling captured prey (Wourms

$61 \&$ Wasserman, 1985). Thus, the experimental research available supports the idea that false

62 heads help butterflies to deflect attacks away from their less vulnerable end (Wourms \&

63 Wasserman, 1985; Sourakov, 2013).

However, these experimental studies have some limitations. Sourakov's (2013) sample

size was very small and the control group differed in a number of morphological and behavioural aspects besides the absence of a false head. Wourms \& Wasserman (1985) recognized that the wing shape of $P$. rapae is different from that of "false-head" Lycaenidae and that some of the behaviours associated with the functioning of false heads are absent in this species. Furthermore, although these studies support the deflecting function of false heads, visually guided predators of butterflies exhibit a variety of sensory capabilities and employ different hunting strategies, and it is not clear if false heads are useful against all them.

Salticid spiders and birds are active hunters that are probably able to use fine details to identify and attack butterflies, while sit-and-wait predators, such as mantises, appear to recognize prey by assessing a number of general features in objects found in the environment (Kral, 2012; Prete et al., 2013). According to behavioural and electrophysiological studies (reviewed in Prete at al., 2013), the main features used by mantises include the size of the object, contrast with the background, leading edge length, speed and movement pattern. Thus, considering the last two features, we hypothesize that the movement of false antennae (i.e., the "tails" present in the border of the anal angle of the hindwings) deflects mantis attacks to a less vulnerable area and increases the probability of escape. We tested this idea by measuring the effects of experimentally ablating the hindwing tails of the false head butterfly Callophrys xami (Lycaenidae: Techlinae) (Fig. 1) on the probability of exhibiting hindwing back-and-forth 
83 movement, and on the probability of being attacked and captured by female mantises

84 (Stagmomantis limbata).

85

\section{MATERIALS AND METHODS}

87

Experimental butterflies were raised from eggs laid by three females collected in the Pedregal de San Ángel Ecological Reserve (PSAER) of the Universidad Nacional Autónoma de México (UNAM), located in the main campus of the UNAM in the South of Mexico City. Callophrys $x a m i$ is a multivoltine "false head" butterfly whose main food plant in the collection site is Echeveria gibbiflora DC (Crassulaceae). Rearing methods followed Jiménez \& Soberón (19881989).

The predators used in the experiment were adult females (males did not attack butterflies in pilot tests) of the mantis Stagmomantis limbata, a species living in the PSAER and, therefore, a potential natural predator of C.xami. Some of the females were the offspring of a female collected in the PSAER, whose nymphs were maintained individually in $1 / 2 \mathrm{~L}$ plastic containers until the fourth instar and afterwards in 1 L containers. Nymphs from instars 1 to 3 were fed Drosophila nubin ad libitum every other day, and afterwards with Achaeta domesticus crickets. The rest of the female mantises used were donated as adults by the Unidad de Manejo Ambiental Yolkatsin (México), where a colony of mantises raised in captivity has been maintained during several generations. These mantises were also fed Drosophila from instar 1 to 3 , and $A$. domesticus afterwards. Thus, before our experiment, none of the mantises had been in contact with butterflies. All insects were maintained at ambient temperature under a $12 \mathrm{~h}$ dark-12 h light photoperiod in the insectary of the Instituto de Ecología (UNAM) located besides the PSAER. 
the hindwing tails were ablated (Fig. 1b), whereas in the control group the wings remained intact

107 (Fig. 1a). Hindwing tails ablation was achieved by first introducing the butterflies in a $-20^{\circ} \mathrm{C}$

108 freezer until they were immobile (between 2 and $5 \mathrm{~min}$ ), then the tails were cut out with micro-

109 scissors (Iris Scissors, Bioquip ${ }^{\text {TM}}$ ). Manipulation of each butterfly lasted approximately $2 \mathrm{~min}$.

110 Control individuals were also introduced in the freezer and manipulated for a similar amount of

111 time as experimental butterflies. Twenty-six butterflies of both sexes were attacked thus

112 producing experimental data (14 males: 8 control, 6 experimental; 12 females: 6 control, 6

113 experimental; see Appendix).

Twenty-four female mantises were used, but five never attacked. Twelve mantises that

attacked were used just once (six with experimental and six with control butterflies) and seven were used twice (five first with a control and then with an experimental butterfly, and two first with an experimental and then with a control butterfly). Mantises used twice had a time interval between trials of at least two weeks thus reducing possible learning effects. The fact that only two of the seven mantises captured both butterflies and that other four captured the first but failed capturing the second butterfly, suggests learning had no effect on our results. To increase the probability of attack, mantises were starved three days before being exposed to a butterfly. Butterflies were individually exposed to one mantis in a glass chamber measuring 29.5 $\mathrm{cm} \times 25 \mathrm{~cm} \times 9.5 \mathrm{~cm}($ length $\times$ height $\times$ width $)$, with one of the two largest $(29.5 \mathrm{~cm} \times 25 \mathrm{~cm})$

124 sides covered with white Styrofoam. A Sony Handycam HDR-SR1was used to film most of the 125 trials (23 out of 26). The room where the experiments were carried out was illuminated with two 30W white fluorescent tubes (Philips ${ }^{\text {TM }}$ Slim line LDD F48T8/TL865) located at a diagonal

127 distance from the chamber (i.e., they were not directly above it) of $2.5 \mathrm{~m}$ and $3.6 \mathrm{~m}$, respectively. 
128 The mantis was introduced to the experimental chamber two hours before each trial. Afterwards, 129 the butterfly was gently introduced in the chamber in a position as far as possible from the 130 mantis. A trial was discarded if the mantis failed to attack the butterfly within $5 \mathrm{~min}$. If the 131 mantis attacked within five minutes after the introduction of the butterfly, we recorded the result 132 (i.e. butterfly captured or escaped) and finished the trial. We allowed just one attack.

\section{RESULTS}

We staged 22 control and 22 experimental interactions between a mantis and a butterfly.

Twenty-six butterflies (59.1\%) were attacked. The butterflies were attacked when they were walking, perching after walking or after landing; in one case the butterfly was detected after stepping on one leg of the mantis. The number of butterflies attacked (Fig. 2a) was statistically independent of the presence of hindwing tails (Chi squared $=0.38, P=0.54, g l .=1$ ). The number of attacked butterflies displaying hindwing movements (that presumably allow the hindwing tails to mimic the movement of antennae) during the interaction with a mantis (Fig. 2b) was statistically independent of the presence of hindwing tails (Fisher's exact test, $P=0.27$ ). the presence of hindwing tails (Fisher's exact test, $P=0.70$ ). Attacks directed to the rear end of the butterfly resulted in less captures than those directed to other body parts (lateral and frontal attacks): five out of six butterflies escaped when attacked in the rear end, in contrast to four out of 17 attacks directed to other parts (Fisher's exact test, $P=0.018$ ). (We have not videos of three

148 interactions, one of them of a control butterfly that escaped.) However, two of the five failed attacks directed to the rear end involved butterflies with their hindwing tails ablated.

150 Furthermore, only in one case the mantis directed the attack towards the "false head" despite the 
151 real head of the (control) butterfly was closer to the head and front legs of the mantis (see

152 interaction between butterfly 127 and mantis 17 in seconds 27 to 43 of Video). In the other four

153 failed attacks, the rear end of the butterfly was closer to the head and front legs of the mantis (see 154 Video).

155

\section{DISCUSSION}

157 In false head butterflies, the tails present in the anal angle of the hindwings are considered to 158 mimic the antennae of the real head, a hypothesis consistent with the peculiar back-and-forth 159 movements of the closed hindwings that apparently aid mimicking the movement of the real 160 antennae (Robbins, 1980; López-Palafox et al., 2015). This idea led us to predict that the success 161 in escaping a mantis attack would decrease in butterflies with "false antennae" experimentally 162 ablated. Nevertheless, our experiment failed to reveal an advantage of possessing hindwings 163 tails. The presence of hindwings tails in perching C. xami butterflies had no statistically significant effect on the probability of surviving an attack from a mantis that is possibly a natural 165 predator.

Furthermore, although the absolute difference in the proportion of butterflies escaping an 167 attack was in the predicted direction (Fig. 2), direct evidence of improved deception due to the 168 presence of false antennae is weak. In one case, the mantis was apparently deceived into 169 attacking the rear end (false head) of a butterfly with false antennae, judging from the fact that

170 the mantis' head and front legs were closer to the real head (see interaction between butterfly 127 171 and mantis 17 in seconds 27 to 43 of Video). However, in the other four failed attacks directed to 172 the rear end, the back of the butterfly was closer to the front legs of the mantis, suggesting that 
173 the mantis was not deceived into attacking that part. Furthermore, two of these four failed attacks

174 involved butterflies with hindwing tails ablated. no function in this species, but being present because they were inherited from their phylogenetic ancestors. We cannot discard this possibility, but phylogenetic inertia seems unlikely considering that in Theclinae (the diverse subfamily including C. xami) false head components evolve rapidly (Robbins, 1981). Second, hindwing tails could be involved in a different function, such as in courtship behaviour or flight manoeuvrability. These alternatives deserve further study. Finally, hindwing tails could improve the deceiving effect of "false heads" (i.e. act as "false antennae") against predators different from mantises, such as birds that detect their prey by using fine details of the wings and actively, and rapidly, approach it from a relatively long distance. In contrast, against a mantis, a predator that relies on crypsis and has a sit-and-wait strategy that allows more time to observe the prey at close range, hindwing tails could be useless. In fact, our observations suggest that $S$. limbata cryptic appearance and behaviour is quite successful against $C$. xami since in many cases the attacked butterflies approached the mantis (in one case was the butterfly was detected because stepped over a mantis leg). Furthermore, the back and forth movements of the closed hindwings, that presumably permit the "false antennae" mimic the movements of real antennae (Robbins, 1980; López-Palafox et al., 2015), possibly have a negative effect because they attract the attention of the mantis (Prete et al., 2013).

Although our observations show that in many cases mantises did not direct their attacks towards the "false head", and that many attacks resulted in successful capture of butterflies (16 out of 26 in our experiment), our study also indicates that at least some aspects of the "false head" help C. xami survive some mantis attacks, supporting the notion that they are adaptations 
196

197

198

199

200

201

202

203

204

205

206

207

208

209

210

211

212

213

214

215

against predators (Robbins, 1980; Cordero, 2001; Sourakov, 2013). Five out of six butterflies

that were attacked in the "false head" zone were able to escape. In two of these cases (one

control and one with hindwing tails ablated), the mantis teared small pieces of wing from the false head area (see interactions between butterfly 92 and mantis 16 in seconds 21 to 28 , and between butterfly 129 and mantis X in seconds 59 to 62 of Video), an observation consistent with the idea that the "false head" area breaks-off easily (Robbins, 1980). Thus, our observations indicate that escaping from an attacking mantis depends on several factors, such as the ability to take flight rapidly (see Video) and the specific part of the wings grabbed by the mantis.

\section{ACKNOWLEDGEMENTS}

This study is part of Tania Guadalupe López Palafox (TGLP) Master in Sciences thesis in the Posgrado en Ciencias Biológicas, Universidad Nacional Autónoma de México. We thank Drs. Marcela Osorio, Atilano Contreras, Matthew Lin, Claudio Lazzari and Robert Robbins for valuable commentaries, and Raúl Martínez Becerril and Isabel Vargas Fernández for technical support. We thank Luis Antonio Cedillo Vázquez (UMA Yolkatzin) and Eric Martínez Luque for providing mantises. CC deeply loves María.

\section{REFERENCES}

Cordero C. 2001. A different look at the 'false head' of butterflies. Ecological Entomology 26:106-108. DOI: 10.1046/j.1365-2311.2001.00287.x. 
216 Ellers J, Boggs CL. 2003. The evolution of wing color: male mate choice opposes adaptive

217

218

219

220

221

222

223

224

225

226

227

228

229

230

231

232

233

234

235

236

wing color divergence in Colias butterflies. Evolution 57:1100-1106. DOI:

10.1111/j.0014-3820.2003.tb00319.x.

Finkbeiner SD, Briscoe AD, Reed RD. 2014. Warning signals are seductive: relative contributions of color and pattern to predator avoidance and mate attraction in Heliconius butterflies. Evolution 68:3410-3420. DOI: 10.1111/evo.12524.

Jiménez G, Soberón J. 1988-1989. Laboratory rearing of Sandia xami xami (Lycaenidae: Eumaeini). Journal of Research on the Lepidoptera 27:268-271. Available at: http://lepidopteraresearchfoundation.org/journals/27/PDF27/27-259.pdf.

Kemp DJ, Rutowski RL. 2011. The role of coloration in mate choice and sexual interactions in butterflies. Advances in the Study of Behavior 43:55-92. Available at: http://dx.doi.org/10.1016/B978-0-12-380896-7.00002-2

Kral K. 2012. The functional significance of mantis peering behavior. European Journal of Entomology 109:295-301. DOI: 10.14411/eje.2012.039

López-Palafox TL, Luis-Martínez A, Cordero C. 2015. The movement of "false antennae" in butterflies with "false heads". Current Zoology 61:758-764. DOI: https://doi.org/10.1093/czoolo/61.4.758.

Monteiro A, Prudic KL. 2010. Multiple approaches to study color pattern evolution in butterflies. Trends in Evolutionary Biology 2:e2. DOI: 10.4081/eb.2010.e2.

Prete FR, Theis R, Dominguez S, Boguee W. 2013. Visual stimulus characteristics that elicit tracking and striking in the praying mantises Parasphendale affinis, Popa spurca and 
Robbins R. 1980. The Lycaenid "false head" hypothesis: Historical review and quantitative analysis. Journal of the Lepidopterists' Society 34:194-208. Available at: http://images.peabody.yale.edu/lepsoc/jls/1980s/1980/1980-34(2)194-Robbins.pdf.

Robbins R. 1981. The "False head" hypothesis: Predation and wing pattern variation in Lycaenid butterflies. American Naturalist 118:770-775. Available at: http://www.journals.uchicago.edu/doi/abs/10.1086/283868.

Ruxton GD, Sherratt TN, Speed MP. 2004. Avoiding attack. The evolutionary ecology of crypsis, warning signals, and mimicry. Oxford: Oxford University Press.

Sourakov A. 2013. Two heads are better than one: false head allows Calycopis cecrops (Lycaenidae) to escape predation by a jumping spider, Phidippus pulcherrimus (Salticidae). Journal of Natural History 47:1047-1054. DOI: http://dx.doi.org/10.1080/00222933.2012.759288.

Wickler W. 1968. El mimetismo en las plantas y los animales. Madrid: McGraw-Hill: Biblioteca para el Hombre Actual No. 29.

Wourms W, Wasserman FE. 1985. Butterfly wing markings are more advantageous during handling than during the initial strike of an avian predator. Evolution 39:845-851. DOI: $10.2307 / 2408684$ 


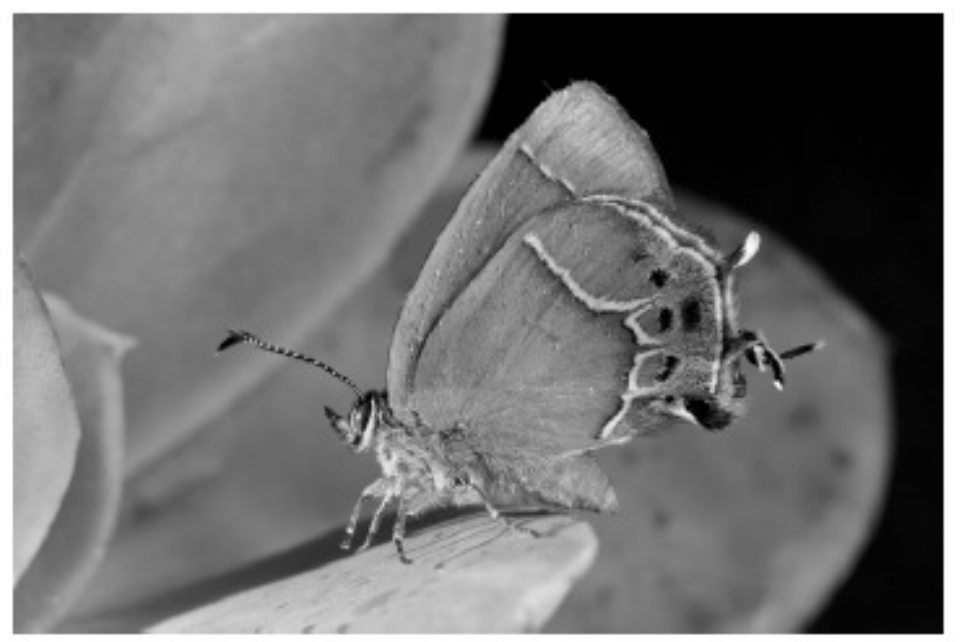

258 Figure 1 Callophrys xami (a) with hindwing tails intact (control) and (b) with hindwing tails by Raúl Iván Martínez. 
a) Attacked?

267

268

269

270

271

272

273

274

275
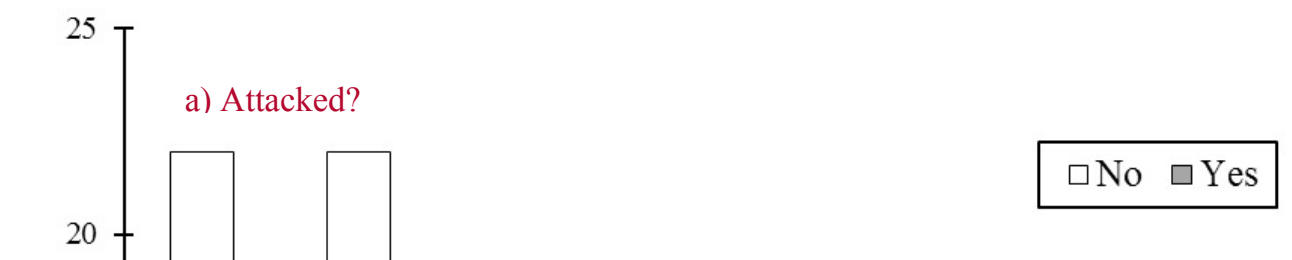

68

Figure 2 Experimental ablation of hindwing tails ("false antennae") in the "false head" butterfly Callophrys xami and its effect on interactions with female mantis (Stagmomantis limbata). Control butterflies were manipulated in the same way as experimental butterflies but their hindwing tails were not ablated. (a) Number of butterflies attacked (gray) or ignored (white). (b) Number of butterflies that performed hindwing movements (gray) or not (white) before being attacked. (c) Number of butterflies escaping (gray) or being captured (white). None

284 of the differences between control and experimental groups were statistically significant (see text). 
287 APPENDIX

288 Raw data from the experiment on the effect of ablation of butterfly (Callophrys xami)

289 hindwing tails ("false antennae") on hindwing movement (HWM) and capture by female

290 mantis (Stagmomantis limbata). Control butterflies were manipulated in the same way as

291 experimental butterflies but their hindwing tails were not ablated.

\begin{tabular}{ccccc}
\hline Treatment & Mantis code & Butterfly & HWM & Result \\
\hline Control & 4 & $37-\mathrm{F}$ & No & Captured \\
Control & 11 & $14-\mathrm{M}$ & No & Captured \\
Control & $\mathrm{T}$ & $106-\mathrm{F}$ & No & Captured \\
Control & $\mathrm{X}$ & $117-\mathrm{F}$ & No & Captured \\
Control & 1 & $1-\mathrm{M}$ & Yes & Captured \\
Control & 11 & $126-\mathrm{M}$ & Yes & Captured \\
Control & 15 & $90-\mathrm{F}$ & Yes & Captured \\
Control & $\mathrm{Z}$ & $78-\mathrm{F}$ & Yes & Captured \\
Control & 5 & $39-\mathrm{M}$ & No & Escaped \\
Control & 13 & $94-\mathrm{M}$ & No & Escaped \\
Control & 1 & $38-\mathrm{M}$ & Yes & Escaped ${ }^{\mathrm{b}}$ \\
Control & 12 & $86-\mathrm{M}$ & Yes & Escaped \\
Control & 16 & $92-\mathrm{M}$ & Yes & Escaped \\
Control & 17 & $127-\mathrm{F}$ & Yes & Escaped \\
Tails ablated & 14 & $70-\mathrm{M}$ & No & Captured \\
Tails ablated & 14 & $102-\mathrm{F}$ & No & Captured \\
Tails ablated & $1 \mathrm{E}$ & $68-\mathrm{M}$ & No & Captured \\
Tails ablated & $2 \mathrm{E}$ & $60-\mathrm{M}$ & No & Captured \\
Tails ablated & $\mathrm{A}$ & $101-\mathrm{F}$ & No & Captured \\
Tails ablated & $\mathrm{T}$ & $128-\mathrm{F}$ & No & Captured \\
Tails ablated & 7 & $33-\mathrm{F}$ & Yes & Captured \\
Tails ablated & 15 & $119-\mathrm{M}$ & Yes & Captured \\
Tails ablated & 10 & $8-\mathrm{M}$ & No & Escaped \\
Tails ablated & 6 & $42-\mathrm{F}$ & No & Escaped \\
Tails ablated & 5 & $18-\mathrm{M}$ & Yes & Escaped \\
Tails ablated & $\mathrm{X}$ & $129-\mathrm{F}$ & Yes & Escaped \\
\hline & & &
\end{tabular}

${ }^{a} \mathrm{M}$ : male, $\mathrm{F}$ : female. ${ }^{\mathrm{b}}$ Interaction not recorded in video. 